\title{
Intermittency Route to Strange Nonchaotic Attractors
}

\author{
Awadhesh Prasad, Vishal Mehra and Ramakrishna Ramaswamy \\ School of Physical Sciences, Jawaharlal Nehru University, New Delhi 110067, India
}

(November 7, 2018)

\begin{abstract}
Strange nonchaotic attractors (SNA) arise in quasiperiodically driven systems in the neighborhood of a saddle node bifurcation whereby a strange attractor is replaced by a periodic (torus) attractor. This transition is accompanied by Type-I intermittency. The largest nontrivial Lyapunov exponent $\Lambda$ is a good order-parameter for this route from chaos to SNA to periodic motion: the signature is distinctive and unlike that for other routes to SNA. In particular, $\Lambda$ changes sharply at the SNA to torus transition, as does the distribution of finite-time or $N$-step Lyapunov exponents, $\mathrm{P}\left(\Lambda_{N}\right)$.
\end{abstract}

Typeset using REVTEX 
Strange nonchaotic attractors (SNAs), which are commonly found in quasiperiodically forced systems, are geometrically strange - they are properly described by a fractal dimension - but the largest nontrivial Lyapunov exponent $\Lambda$ is negative, implying nonchaotic dynamics.

Since they were first described [1], and shown to be generic in quasiperiodically driven nonlinear systems [2], a number of characteristics of SNAs have been studied, including the important question of how they are created. Three main mechanisms or scenarios for the creation of SNAs have been advanced. Heagy and Hammel [3] identified the birth of a SNA with the collision between a period-doubled torus and its unstable parent. This mechanism is analogous to the attractor-merging crisis that occurs in chaotic systems [4]. Kaneko [5, 6] has described the "fractalization" of a torus, namely the increasing wrinkling of tori which leads to the appearance of a SNA without any interaction with a nearby unstable periodic orbit. This route to SNA (and eventually to chaos) has also been observed in several systems. Yalçinkaya and Lai [7] have recently shown a third route to SNAs via a blowout bifurcation [8], namely through the loss of transverse stability of a torus.

We describe a new mechanism for the creation of SNAs. As a function of driving parameter, a strange attractor disappears and is eventually replaced by a 1-frequency torus through an analogue of the saddle-node bifurcation. In the vicinity of this crisis-like phenomenon [4] the attractor is strange and nonchaotic. We show that the dynamics at this transition is intermittent, and the scaling behaviour is characteristic of Type I intermittency [9]. Furthermore, the signature of the transition in $\Lambda$ (or in the distribution of finite-time Lyapunov exponents) is distinctive and very different from the routes to SNA that have been hitherto discussed. The present mechanism is general and is likely to be operative in any quasiperiodically driven system.

For definiteness, we illustrate our results using the quasiperiodically forced logistic $\operatorname{map}[3$

$$
x_{n+1}=\alpha\left(1+\epsilon \cos \left(2 \pi \phi_{n}\right)\right) x_{n}\left(1-x_{n}\right)
$$




$$
\phi_{n+1}=\phi_{n}+\omega \quad(\bmod 1) .
$$

where we take $\omega=(\sqrt{5}-1) / 2$, the golden mean, and $2 \leq \alpha \leq 4$. This system is convenient to study since the phenomenology is smoothly related to that of the logistic map in the limit of $\epsilon \rightarrow 0$. Since the driving term is multiplicative, it is clear that the motion will remain bound if $\alpha\left(1+\epsilon \cos \left(2 \pi \phi_{n}\right)\right) \in[0,4]$, and thus for any $\alpha$, the largest value of $\epsilon$ allowed is $4 / \alpha-1$. It is preferable to work with the rescaled driving parameter, $\epsilon^{\prime}=\epsilon /(4 / \alpha-1)$ since this scales the region of interest in parameter space to $0 \leq \epsilon^{\prime} \leq 1$.

Fig. 1 is a phase-diagram of the system showing the different possible dynamical behaviours - periodic, strange nonchaotic and chaotic attractors, corresponding to the symbols P, S and C. The different phases are characterised through the largest nonzero Lyapunov exponent $\Lambda=\lim _{N \rightarrow \infty} \frac{1}{N} \sum_{i=1}^{N} \ln \left|\left(1-2 x_{i}\right) \alpha\left(1+\epsilon \cos \left(2 \pi \phi_{i}\right)\right)\right|$, which we calculate typically from $10^{6}$ iterations of the map, as well as the phase-sensitivity exponent [10]. The logistic map with additive driving [6] has identical behaviour.

SNAs occur along the boundary of the chaotic regions [11], when $\Lambda$ takes small negative values. In the $\alpha-\epsilon^{\prime}$ plane, the regions of $\mathrm{P}, \mathrm{S}$ and $\mathrm{C}$ behaviour can be interwoven. In particular, there are two distinct chaotic phases: the regions marked $\mathrm{C}_{1}$ and $\mathrm{C}_{2}$ which are separated by a narrow tongue where the motion is periodic. The behaviour of $\Lambda$ as a function of $\alpha$ for $\epsilon^{\prime}=1$, namely along the upper edge of Fig. 1, is shown in Fig. 2(a). The nonmonotonicity of $\Lambda$ as a function of $\alpha$ is typical, and several dynamical transitions can be described: $\mathrm{P} \rightarrow \mathrm{S}, \mathrm{S} \rightarrow \mathrm{C}$, and $\mathrm{C} \rightarrow \mathrm{S}$ (note that periodic motion here corresponds to a n-frequency torus, with $\mathrm{n}=1,2$ or 4 ; higher $\mathrm{n}$ such as 3, 6, 8 etc. can also be observed in small windows of parameter space).

The intermittency route to SNA that we describe in this work occurs along the boundary of the chaotic region $\mathrm{C}_{2}$ and the periodic region $\mathrm{T}$ on its right, namely at the point marked I in Figs. 1 and 2a. An enlarged view is shown in Fig. 2b where the bars indicate the variance in the Lyapunov exponent estimated from several computations. There is a transition from a chaotic attractor to a SNA at $\alpha \approx 3.405802$ with $\Lambda$ changing linearly [12] through zero. The 
intermittent transition from the SNA to a torus - the phenomenon described here - occurs at $\alpha=\alpha_{c} \equiv 3.4058088 \ldots$. . At this transition, the distinctive signature is an abrupt change in the dependence of $\Lambda$ on the parameter $\alpha$, as well as a concurrent marked reduction in the variance in $\Lambda$ (see Fig. $2 \mathrm{~b}$; the bars have been computed from 50 samples of $10^{5}$ steps). The disappearance of the SNA or the chaotic attractor is accompanied by intermittent dynamics, which can be conveniently studied by co-evolving two trajectories with identical $\left(x_{0}, \phi_{0}\right)$ and $\epsilon^{\prime}$, with different $\alpha$ : since the angular coordinate remains identical, the distance between the trajectories is simply the difference in the $x_{n}$ 's. We find that the time between bursts shows the scaling $\tau \sim\left(\alpha_{c}-\alpha\right)^{-\theta}$, with $\theta \approx 1 / 2$ (the numerical value we obtain is $0.52 \pm 0.04$ ). This transition to an intermittent SNA (Fig. 2c) occurs all along the right boundary of $\mathrm{C}_{2}$. In the $\alpha-\epsilon$ plane, this is a region of nearly constant $\epsilon$.

The abrupt death of the strange nonchaotic attractor is through a quasiperiodic analogue of the saddle-node bifurcation where a period 1 orbit is born. Consider a sequence of maps that are rational approximations to Eq. (1) by setting $\omega=\omega_{k}=F_{k} / F_{k+1}$, where $F_{k}$ is the $k$ th Fibonacci number. The $F_{k+1}$ th iterate of the map is a function of $x$ alone (since $\left.\phi_{F_{k+1}} \equiv \phi_{0}\right)$. This allows for the construction of a bifurcation diagram as a function of $\alpha$ for fixed $\epsilon$. The case of $k=2$, namely $\omega=1 / 2$ has been studied earlier by Sanju and Varma [13] as the period-2 modulated logistic map. Between the first period doubling bifurcation and the 2-band merging crisis, the attractor of the (unmodulated) logistic map consists of two branches. (For the logistic map, these bifurcations occur at $\alpha=3$ and $\alpha \approx 3.678857 \ldots$, respectively). The effect of period -2 modulation is to split the two branches of the attractor, by shifting one branch to higher values of $\alpha[13$. The extent to which the two branches are shifted relative to one another depends on the value of $\epsilon$. The single attracting orbit of period -2 in the unmodulated map becomes two separate attracting orbits for small values of the modulation; and depending on how the branches are shifted, namely on the strength of the modulation, two very different attractors - for example chaotic and periodic - can coexist. For sufficiently large $\epsilon$ the two branches can in fact become disjoint, namely the periodic orbit of one branch of the attractor is shifted beyond the 2-band merging point (see 
Fig. 2 of Ref. [13]). When this happens, the periodic attractor appears as at a saddle-node bifurcation, giving rise to a single periodic attracting orbit of period 1 .

It can easily be verified that this mechanism operates with small modifications for the higher rational approximations $\omega_{k}$; this is a case of period $-F_{k+1}$ modulation [13]. Thus, in the limit of $\omega_{k} \rightarrow \omega=(\sqrt{5}-1) / 2$, we suggest that the disappearance of the chaotic attractor and the SNA is through this analogue of a saddle-node bifurcation.

Viewed as a function of decreasing $\alpha$, this transition shares some of the features of a widening crisis in unforced systems [4, 14, 15]. The discontinuous change of $\Lambda$ at a saddlenode bifurcation is softened by the quasiperiodic forcing, and for $\alpha<\alpha_{c}$, the Lyapunov exponent shows the scaling (see Fig. 3)

$$
\Lambda-\Lambda_{c} \sim\left(\alpha_{c}-\alpha\right)^{\mu}
$$

with the exponent $\mu=0.37 \pm 0.03$ at $\epsilon^{\prime}=1$. To within the quoted error-bar, the same exponent is obtained when the probability density in the burst phase is fit to a power law [14 [16]. The remnants of the old torus which are now embedded in the SNA constitute one of the weakly coupled components of the SNA. These exponents stay approximately constant along the $\mathrm{C}_{2}$ boundary.

There are at least two other mechanisms for the creation of SNAs that are operative in this system. Along the boundary of the chaotic region $\mathrm{C}_{1}$ in Fig. 1 , which appears at the end of the (truncated) period doubling cascades, SNAs that are formed can appear as a result of the collision between a period-doubled torus and its unstable parent [a] . SNAs are also created through the process of fractalization [5] which occurs along the entire left edge of the region $\mathrm{C}_{2}$ (for example the cases studied by Nishikawa and Kaneko [6]). Along the $\mathrm{C}_{1}$ boundary both mechanisms operate and representative points are marked as $\mathrm{B}$ and A respectively in Fig. 2a. A fractalizing torus gets increasingly wrinkled as parameters are varied, and eventually goes chaotic, without any period-doubling bifurcation. These two routes can be differentiated from the geometry of the SNA: in the torus collision mechanism [3], a period $2^{k}$-torus gives rise to a $2^{k-1}$-band SNA, while through fractalization [0.6, 6], the 
result is a $2^{k}$-band SNA.

The intermittent SNA is similar in many respects to a reentrant phase: the SNA so created is structurally distinct from the SNA born through other mechanisms. This distinction also extends to the corresponding tori, and examination of successive iterates of the line $(1 / 2, \phi)$ under the map $f$ of Eq. (I) gives an indication of how this occurs. By an extension of the $\epsilon=0$ case [17], this line will be attracted to the 1 -torus,

$$
\lim _{k \rightarrow \infty} f^{k}(1 / 2, \phi) \rightarrow T
$$

(The attractor of the dynamics, whether periodic, strange nonchaotic or chaotic, is confined in a strip in configuration space, the upper boundary of which is the line $f(1 / 2, \phi)$, and the lower boundary is the infimum of $\left.f^{j}(1 / 2, \phi), j \geq 2\right)$. The convergence behaviour of the line to the attractor, quantified through the phase sensitivity index [10] shows that for smaller values of nonlinearity and driving, the convergence is slower than for higher nonlinearity. Indeed, the torus stabilized at large $\alpha, \epsilon^{\prime}$ (in Fig. 1) is relatively smooth, as opposed to the highly structured and fractalizing torus at smaller values of $\alpha$.

To further characterise this distinction, we examine the distribution of finite-time Lyapunov exponents $P\left(\Lambda_{N}\right)$ in Fig. 4, namely the Lyapunov exponent computed from a $N$-step orbit on the attractor. Although $\Lambda$ is negative, for short times the Lyapunov exponent on a SNA is positive [10]. The distribution $P\left(\Lambda_{N}\right)$ has not been studied in detail, but it is known to have a significant positive tail which does not vanish even for large $N$. For the intermittent SNA, $P\left(\Lambda_{N}\right)$ decays slowly both as a function of the (local) $\Lambda_{N}$ as well as $N$. This distinction is a consequence of the fact that the variance in the $\Lambda$ increases drastically through this transition (in Fig. 2b). In contrast, at the other transitions from a torus to a SNA (or vice-versa), the variance does not change significantly and with increasing $N$, the $P\left(\Lambda_{N}\right)$ becomes essentially gaussian. The difference in the distributions is not visible for $N \sim 50$ (Fig. 4a) while at $N \sim 1000$ the distinct character of the intermittent SNA is clearly seen (Fig. 4b). These observations are consistent with a coarser interweaving of the positive and negative local Lyapunov exponent regions on the intermittent SNA. Furthermore, this 
transition is robust to low-amplitude noise [18], as has been verified by adding a small random component to the $x$ dynamics. The scaling behaviour in $\Lambda$ and the probability density in the burst phase were observed to hold [19] with slightly modified values of the exponent.

Such quasiperiodically driven systems are interesting from a variety of points of view. A potential novel use of SNAs that can be envisaged is for secure communications [20]: two independent SNAs can be easily synchronized since the Lyapunov exponents are negative, while the signals from such systems provide effective masking since they appear erratic owing to the underlying strange geometry.

In summary, in the present Letter we have described a new mechanism for the creation of strange nonchaotic attractors through intermittency, whereby a chaotic attractor is eventually replaced by a quasiperiodic one. In the intermediate region, there are SNAs. The distinctive signature of this transition is a sharp change in the Lyapunov exponent which shows large fluctuations and scaling behaviour on the SNA side of the transition. This mechanism, which is operative at high nonlinearity and large amplitude quasiperiodic forcing, proceeds via a stabilization of chaotic motion and is thus like a reentrant phase transition.

The route to intermittent SNA is a general one. We have studied a variety of related systems [19] and find that the phenomenology is very similar: the phase-diagram that is obtained for the driven logistic map is typical. Intermittent SNAs always occur in analogous regions in parameter space, namely where a tongue of periodic behaviour separates two chaotic areas with differing characteristics [21]. We expect that in situations where the amplitude of quasiperiodic forcing is an conveniently varied parameter for example, this mechanism for the creation of SNAs may be experimentally observed.

This work was supported by grant SPS/MO-5/92 from the Department of Science and Technology, India. We thank Sanju for discussions. 


\section{REFERENCES}

[1] C. Grebogi, E. Ott, S. Pelikan and J.A. Yorke, Physica D 13, 261 (1984)

[2] F.J. Romeiras, A. Bondeson, E. Ott, T.M. Antonsen, and C. Grebogi, Physica D 26, 277 (1987);T. Zhou, F. Moss, and A. Bulsara, Phys. Rev. A, 45, 5394 (1992); S.P. Kuznetsov, A.S. Pikovsky, and U. Feudel, Phys. Rev. E, 51, R1629 (1995)

[3] J.F. Heagy and S.M. Hammel, Physica D 70, 140 (1994)

[4] C. Grebogi, E. Ott, F.J. Romeiras and J.A. Yorke, Phys. Rev. A, 63, 5365 (1987)

[5] K. Kaneko, Prog. Theor. Phys., 71, 1112 (1984)

[6] T. Nishikawa and K. Kaneko, Phys. Rev. E, 54, 6114 (1996)

[7] T. Yalçinkaya and Y.C. Lai, Phys. Rev. Lett., 77, 5039 (1996)

[8] E. Ott and J.C. Sommerer, Phys. Letts. A 188, 39 (1994); P. Ashwin, J. Buescu, and I. Stewart, Phys. Letts. A 193, 126 (1994); Y.C. Lai and C. Grebogi, Phys. Rev. E, 52, R3313 (1995)

[9] Y. Pomeau and P. Manneville, Commun. Math. Phys., 74, 189 (1980)

[10] A. Pikovsky and U. Feudel, CHAOS, 5, 253 (1995); U. Feudel, J. Kurths and A. Pikovsky, Physica D 88, 176 (1995)

[11] O. Sosnovtseva, U. Feudel, J. Kurths and A. Pikovsky, Phys. Letts. A 218, 255 (1996)

[12] Y.C. Lai, Phys. Rev. E, 53, 57 (1996); Y.C. Lai, U. Feudel, and C. Grebogi, Phys. Rev. E, 54, $6070(1996)$

[13] a) Sanju and V. S. Varma Phys. Rev. E, 48, 1670 (1993); b) Sanju and V. S. Varma in Computational aspects in Chaos and Nonlinear dynamics, Eds. G. Ambika and V.M. Nandakumaran, (Wiley Eastern Limited, New Delhi, 1994), pp 201-214.

[14] R. Pompe and R. W. Leven, Phys. Scr. 38, 651 (1988) 
[15] V. Mehra and R. Ramaswamy, Phys. Rev. E, 53, 3420 (1996)

[16] C. Grebogi, E. Ott, and J. Yorke, Physica D 7, 181 (1983)

[17] P. Collet and J.P. Eckmann, Iterated Maps on the Interval as Dynamical Systems, (Birkhäuser, Basel, 1980).

[18] Noise is added by a term of the form $\sigma \xi_{n}$, where $\xi_{n}$ is a uniform random variable in the unit interval. At $\epsilon^{\prime}=1$, for instance, the transition survives for additive noise of amplitude up to $\sigma=10^{-6}$, while at lower $\epsilon^{\prime}$ the transition is robust to even larger $\sigma$ $\approx 10^{-4}$. In other systems with intermittent SNAs the transition may survive to higher noise levels.

[19] A. Prasad, V. Mehra and R. Ramaswamy, Strange Nonchaotic Attractors in the Quasiperiodically forced Logistic Map, Phys. Rev. E, submitted.

[20] K. M. Cuomo and A.V. Oppenheim, Phys. Rev. Lett., 71, 65 (1993); C. Zhou and T. Chen, Europhys. Lett., 38, 261 (1997); R. Ramaswamy, Synchronization of Strange Nonchaotic Attractors, Phys. Rev. E, in press (1997).

[21] We have located intermittent SNAs in a number of systems such as the forced sine-map, as well as higher dimensional examples such as the ring and Hénon maps [11] using this strategy [19]. 


\section{FIGURES}

FIG. 1. Phase diagram for the forced logistic map (schematic) obtained by calculating the Lyapunov exponent as a function of $\alpha$ and the rescaled parameter $\epsilon^{\prime}$ defined as $\epsilon^{\prime}=\epsilon /(4 / \alpha-1)$ in a $100 \times 100$ grid. $\mathrm{P}$ and $\mathrm{C}$ correspond to Periodic (torus) and Chaotic attractors. The shaded region along the boundary of $\mathrm{P}$ and $\mathrm{C}$ corresponds to SNA (marked $\mathrm{S}$ ). The boundaries separating the different regions are convoluted, and regions of SNA and Chaotic attractors are interwoven in a complicated manner. The intermittent SNA is found on the edge of the $\mathrm{C}_{2}$ region marked I.

FIG. 2. (a) The largest Lyapunov exponent $\Lambda$ versus $\alpha$ at the upper edge of the phase plane, namely at $\epsilon^{\prime}=1$ and $2 \leq \alpha \leq 4 . \mathrm{C}_{1}, \mathrm{C}_{2}$ correspond to the two chaotic phases identified in Fig. 1. T denotes periodic motion (tori); SNAs are found along the boundaries of $\mathrm{C}_{1}$ and $\mathrm{C}_{2}$, in the neighbourhood of the points marked A, B and I. (b) An enlarged view of Fig. 2a) near the intermittent transition marked I. The bars indicates the variance in $\Lambda$ computed from 50 samples of length $10^{5}$. (c) The Intermittent SNA at $\alpha=3.405808$ and $\epsilon^{\prime}=1$.

FIG. 3. Scaling of the probability density $\left(p_{B}\right)$ in the burst phase (o) and $\Lambda(\square)$ at the intermittent transition at I, for $\alpha_{c}=3.4058088 \ldots$ and $\Lambda_{c}=-0.0283$ at $\epsilon^{\prime}=1$. The measured exponents are $\approx 0.37$.

FIG. 4. (a) Distribution of $N$-step or local Lyapunov exponents, computed from $\mathrm{N}=50$ step segments of a long trajectory for the intermittent SNA at the point marked I contrasted with SNAs (born through other mechanisms) at the points marked A and B in Fig. 2a. These correspond respectively to I $(+), A(\nabla)$ and $B(\square)$. (b) Same as (a), except that $N=1000$. 


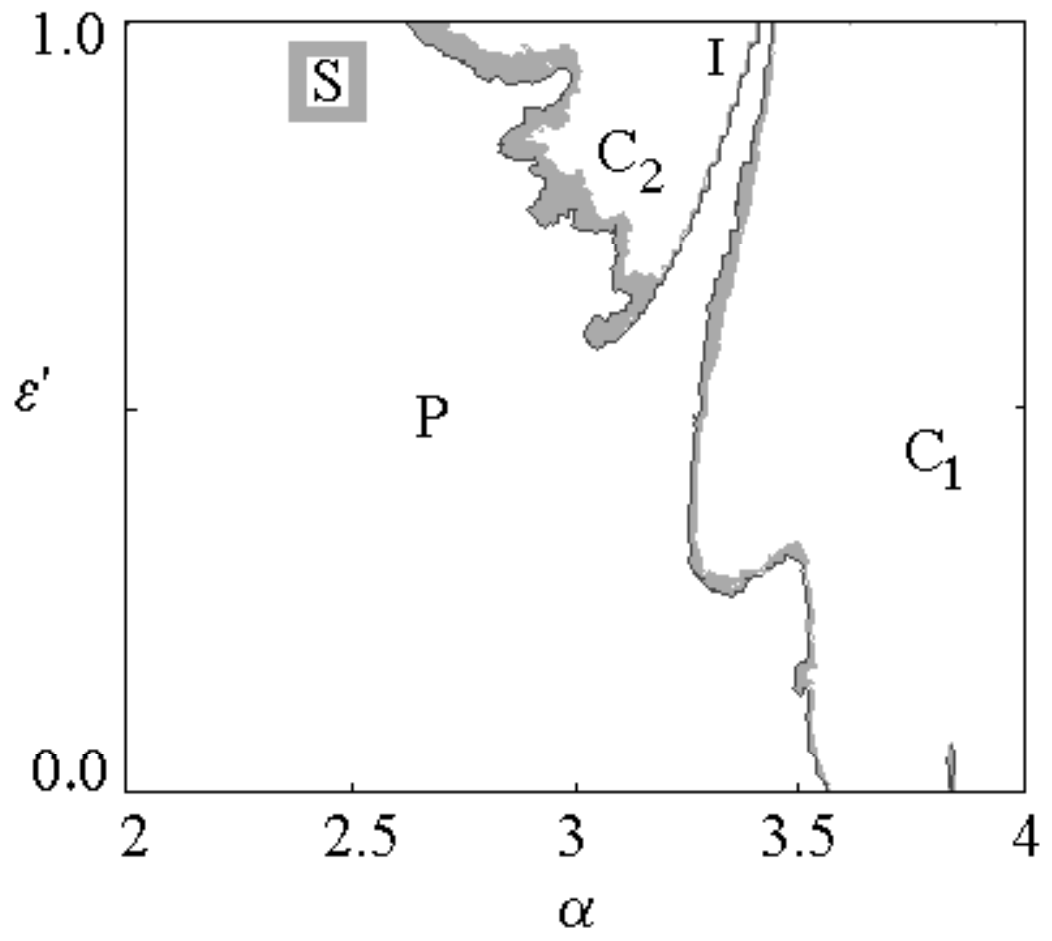




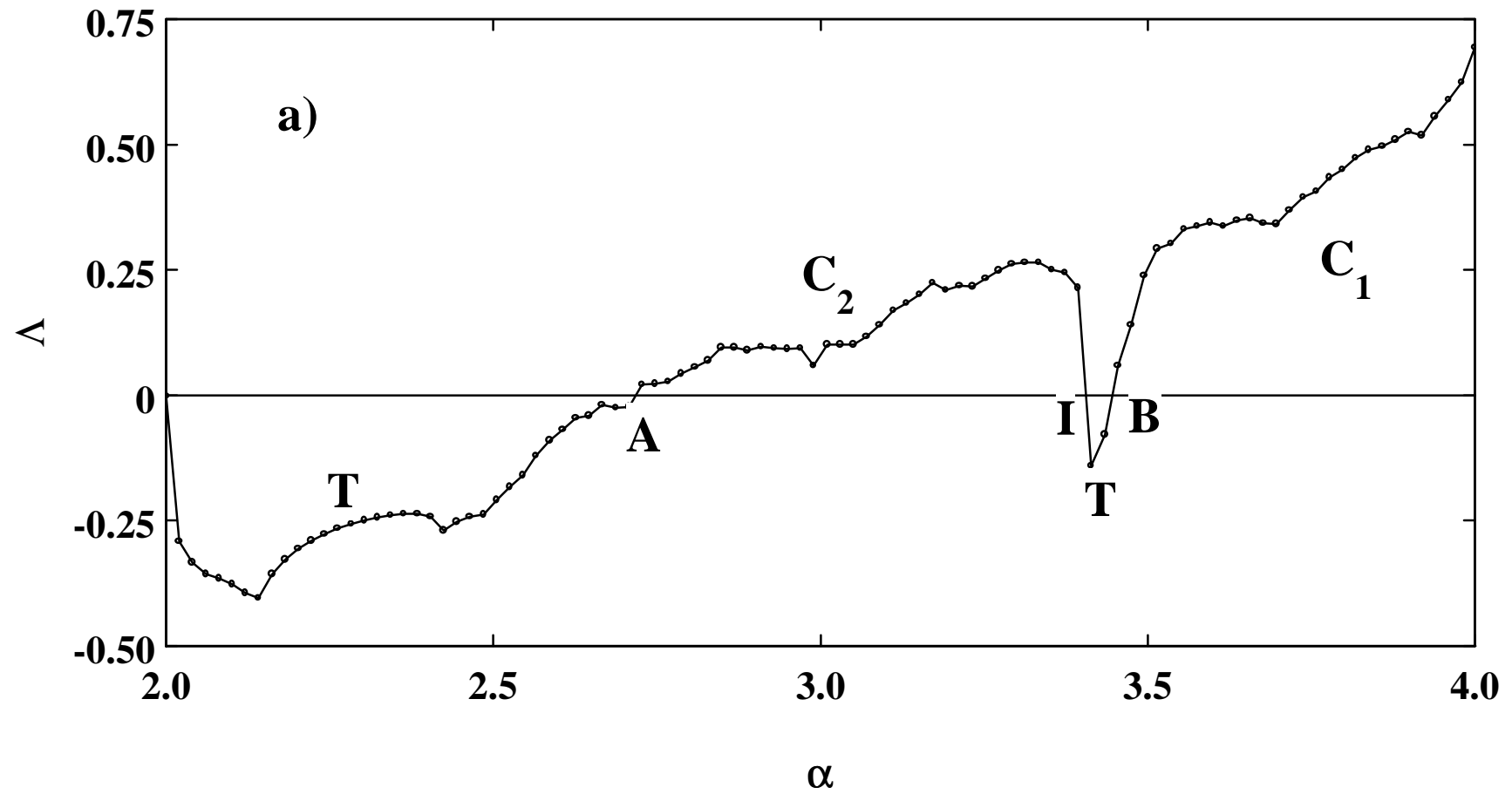




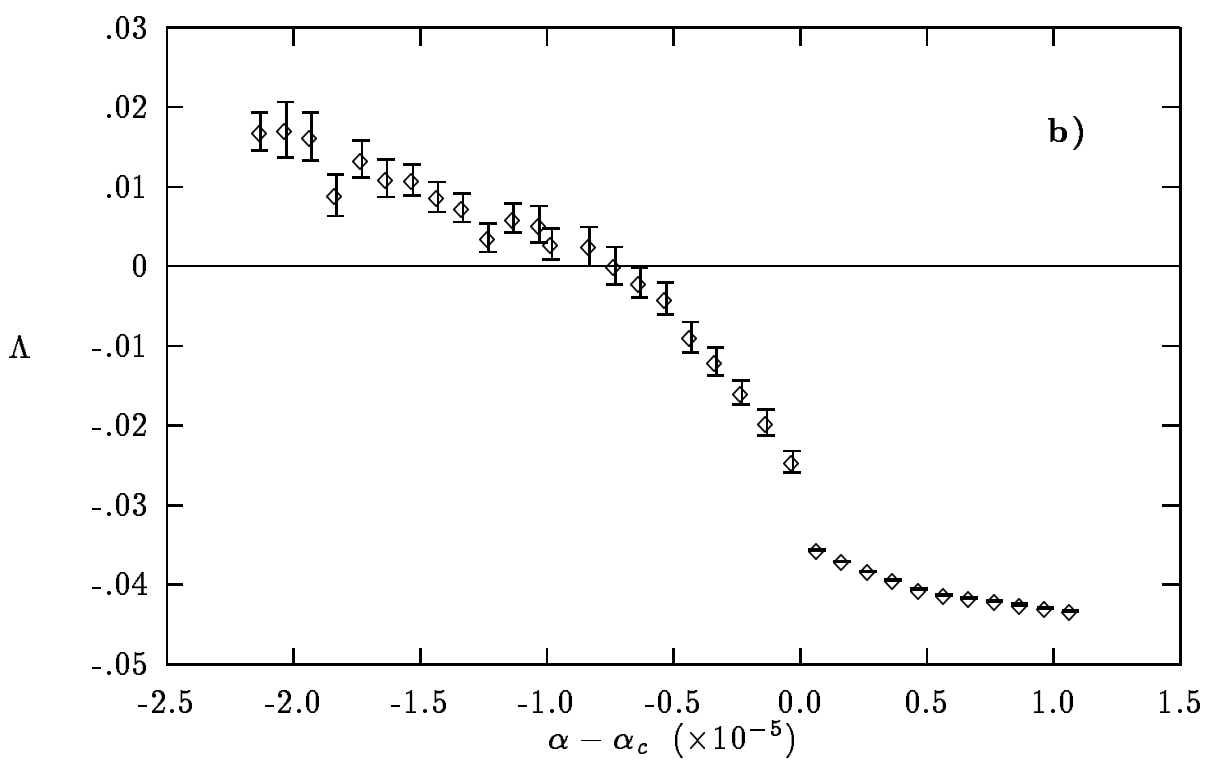




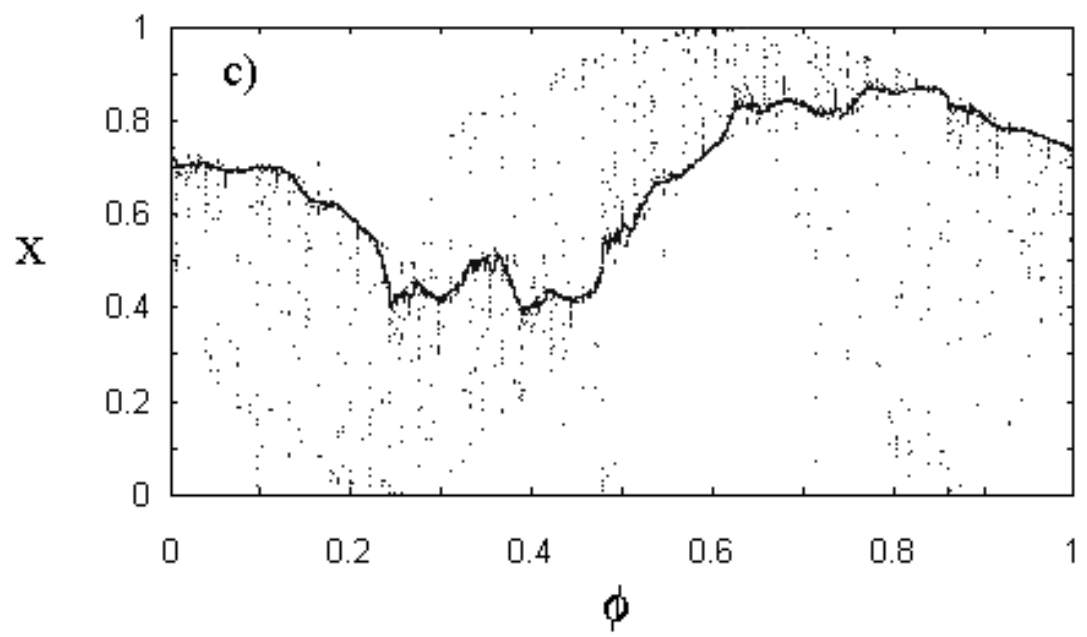




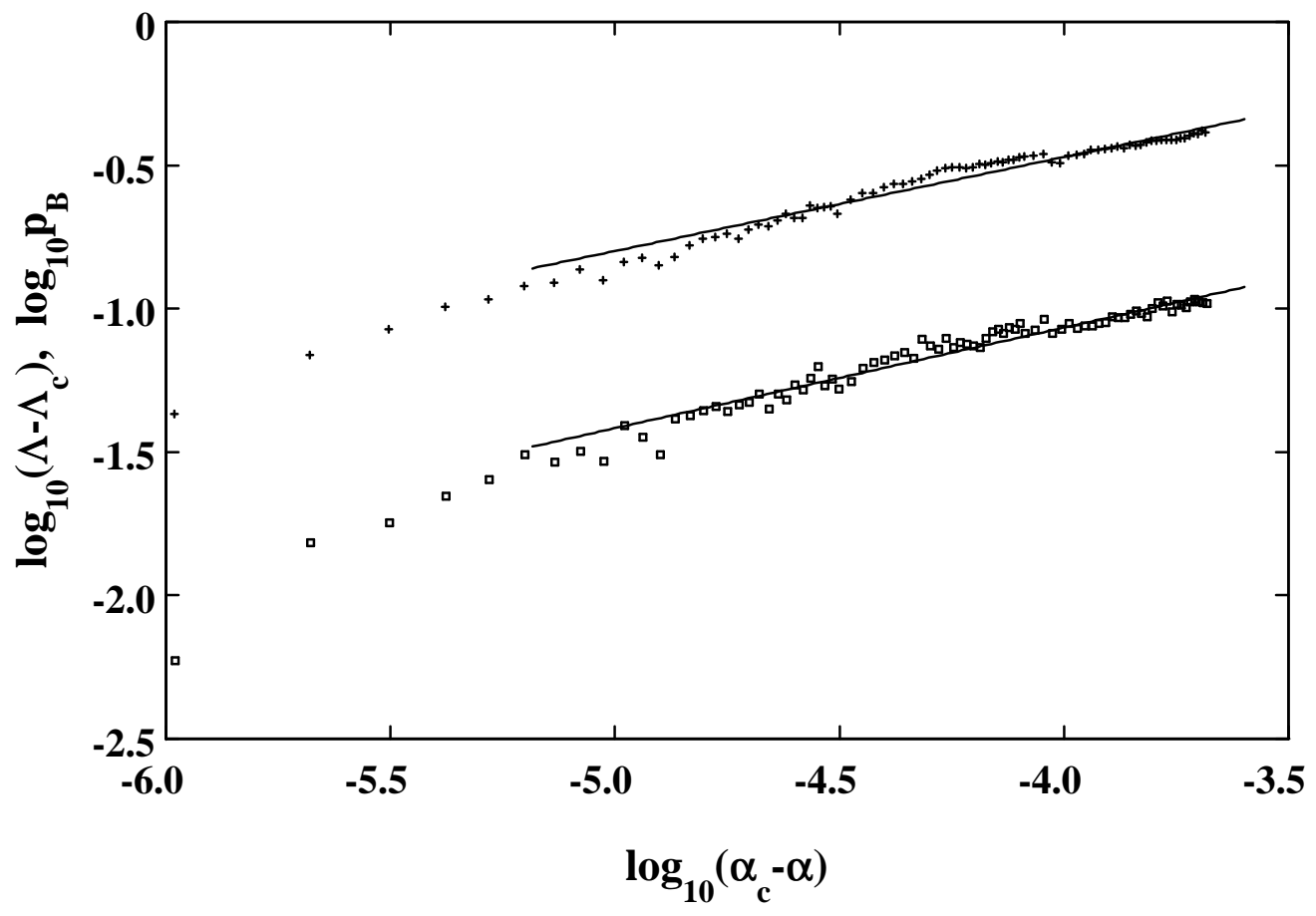



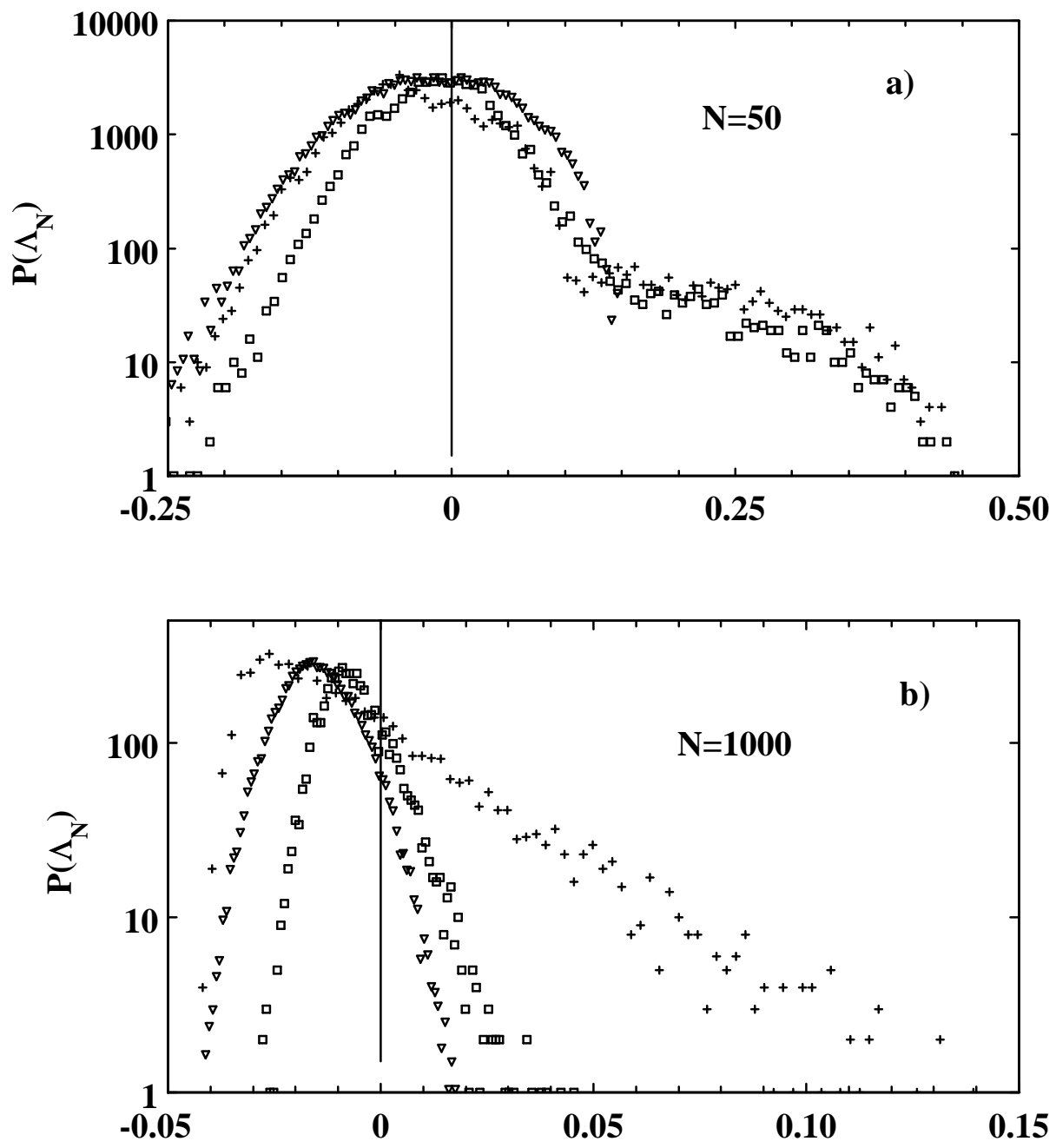

$\Lambda_{\mathrm{N}}$ 